\begin{tabular}{|c|l|}
\hline Title & Numerical simulation of concentration interface in stratified suspension : Continuum-particle transition \\
\hline Author(s) & Y amamoto, Y asufumi; Hisataka, Fumiya; Harada, Shusaku \\
\hline Citation & $\begin{array}{l}\text { International Journal of Multiphase Flow, 73, 71-79 } \\
\text { https://doi.org/10.1016/.jmultiphaseflow.2015.03.007 }\end{array}$ \\
\hline Issue Date & 2015-07 \\
\hline Doc URL & http://hdl.handle.net/2115/66892 \\
\hline Rights & $\begin{array}{l}\text { O2015. This manuscript version is made available under the CC-BY-NC-ND 4.0 license } \\
\text { http://creativecommons.org/icenses/by-nc-nd/4.0/ }\end{array}$ \\
\hline Rights(URL) & http://creativecommons.org/icenses/by-nc-nd/4.0/ \\
\hline Type & article (author version) \\
\hline Information & \\
\hline
\end{tabular}

Instructions for use 


\title{
Numerical simulation of concentration interface in stratified suspension: continuum-particle transition
}

\author{
Yasufumi Yamamoto ${ }^{\mathrm{a}, *}$, Fumiya Hisataka $^{\mathrm{b}}$, Shusaku Harada $^{\mathrm{c}}$ \\ ${ }^{a}$ Department of Mechanical Engineering, Kansai University, 3-35, Yamate-cho 3-chome, \\ Suita, Osaka, 564-8680, Japan \\ ${ }^{b}$ Graduate School of Science and Engineering, Kansai University, 3-35, Yamate-cho \\ 3-chome, Suita, Osaka, 564-8680, Japan \\ ${ }^{c}$ Division of Sustainable Resources Engineering, Hokkaido University, N13W8, Sapporo, \\ Hokkaido 060-8628, Japan
}

\begin{abstract}
Behavior of concentration interfaces, which are apparent interfaces between a suspension and pure liquid, was studied using numerical techniques. Two types of numerical simulations were used to classify whether collective or individual behavior occurred in the ratio of the average particle separation to the wavelength of the fastest growing perturbation. The first is Lagrangian tracking of individual particles in fluid, and the second type is interface tracking of two immiscible continuum phases. These two extremely different approaches represent the dual nature of the concentration interface: immiscible with no interfacial tension and miscible with no diffusion. These results reflect the experimentally-observed behavior of particles, which is both collective and individual. Sealing of the concentration interface by particle-induced flow is crucial to collective motion of suspended particles. A proposed dimensionless parameter describes quantitatively the transition from collective to
\end{abstract}

\footnotetext{
*Corresponding author.

Email address: yamayasu @ kansai-u.ac.jp (Yasufumi Yamamoto)
} 
individual settling of suspended particles.

Keywords: Stratified suspension, Concentration interface, Numerical simulation, Fingering instability

\section{Introduction}

Collective motion of fine particles suspended in a liquid is important not only for engineering disciplines such as mechanical, chemical, civil or environmental ones, but also for other scientific fields as biomechanics or geophysics. Collective motion is observed in various processes and phenomena such as solid-liquid separation (Kynch, 1952), magma flow (Michioka and Sumita, 2005), sea sediment layers, (Carey, 1997), sediment transport in river and ocean (Warrick et al., 2008), and bioconvection (Pedley and Kessler, 1992).

The collective nature of suspended particles is frequently revealed in the mode of gravitational settling of inhomogeneously dispersed suspensions, i.e., the particles are locally suspended in a liquid. Such systems are classified in the following three cases according to the orientation of the boundary between the suspended and nonsuspended regions (this is called the "concentration interface"): (1) the normal vector to the concentration interface (from suspended to nonsuspended region) points up in the vessel, (2) the normal vector points down, and (3) the concentration interface forms a closed surface. The first case corresponds to the well-known case of hindered settling in a vessel, and the concentration interface is called the "sedimentation front." Many studies have focused on the behavior of the sedimentation front from both a macroscopic [i.e., trajectory of the sedimentation front, e.g., (Kynch, 1952)] and a microscopic viewpoint [i.e., hydrodynamic diffu- 
sion near the sedimentation front (Mucha and Brenner, 2003; Bergougnoux et al., 2003), etc. ]. The collective nature of the suspended particles is manifested as a variance in the hindered settling velocity or as self-diffusion due to hydrodynamic interactions in such systems.

The second case is the main subject of the present research wherein the normal to the concentration interface points down. In such a system, the motion of suspended particles is critically influenced by the macroscopic nature of the concentration interface. In other words, the settling velocity of particles is greatly enhanced by the gravitational instability (Rayleigh-Taylor instability) at the concentration interface. Such instabilities and resultant convection caused by particle settling have been studied experimentally (Kuenen, 1968; Hoyal et al., 1999; Parsons et al., 2001; Völtz et al., 2001; Völtz, 2003; McCool et al., 2004; Blanchette and Bush, 2005), theoretically (Burns and Meiburg, 2012; Yu et al., 2013) and numerically (Pan et al., 2001; Chou et al., 2014) in many engineering and science fields.

A similar instability also occurs in the third system, but in a different form. The third system, in which the concentration interface is closed, is called a "suspension droplet." Many studies on suspension droplets have elucidated the collective motion of suspensions (Nitsche and Batchelor, 1997; Machu et al., 2001; Metzger et al., 2007). In these studies, the interfacial instability is found to lead to a characteristic behavior of suspended particles, such as the breakup of droplets in a manner reminiscent of fireworks.

The collective nature of suspended particles is closely associated with the concentration of particles in suspended regions. The suspension droplet is known to essentially maintain a spherical shape for low Reynolds number. 
Previous studies have claimed that this behavior results from the disturbance flow generated by each particle (Stokeslet) sealing the concentration interface, thereby preventing invasion by outside flow. Some researchers have pointed that the sealing ability at the concentration interface of a suspension droplet is determined by the number density in the suspended region (Nitsche and Batchelor, 1997; Metzger et al., 2007). If the number density is small and the Stokeslet does not fully discretize the suspended region, the concentration interface allows penetration by the outside fluid; therefore, some particles may escape from the droplet.

For the second case in which the concentration interface points down, Harada et al. (2012) have experimentally examined the collectivity of settling particles caused by the Rayleigh-Taylor instability. They found that the transition from individual to collective settling of suspended particles is determined by a dimensionless parameter given by the ratio of the interparticle separation to the dominant wavelength of the instability; this ratio is related to the number density of the suspended particles.

Comparing the concentration interface with an ordinary interface reveals the former's peculiar aspects. The concentration interface of a suspension of micron-sized particles is essentially a miscible interface. However, in contrast with a density interface such as a salt-water/fresh-water interface, thermal diffusion is less significant because the particle size is much larger than the size of molecules in the solvent. Conversely, in contrast with an immiscible interface, the interfacial tension of a concentration interface is almost zero, provided the interparticle force is not too large. Therefore, the concentration interface of a suspension with micron-sized particles can be interpreted as an 
ambiguous interface at which the suspended particles are not constrained and are not pushed away from the interface. Fernandez et al. (2001) investigated theoretically the wavelength of the Rayleigh-Taylor instability for both miscible and immiscible interfaces between two fluids. Their results indicate that the dominant wavelength of the instability depends on whether the interface is miscible or immiscible; however, the wavelength of a miscible interface with an infinite Péclet number (i.e., no diffusion) coincides with that of an immiscible interface with infinite capillary number (i.e., no interfacial tension). Therefore, a concentration interface with micron-sized particles can be interpreted as an interface that connects miscible and immiscible interfaces. However, the dual nature of the concentration interface has not been fully explained from the microscopic viewpoint, i.e., how particles which form the interface settle relative to surrounding fluid (as miscible interface) and how the disturbance flow created by each particle prevents the invasion of flow (as immiscible interface).

The present study investigates the Rayleigh-Taylor instability at the concentration interface of a particulate suspension by numerically simulating the resultant collective motion of particles. To examine the dual nature of the concentration interface (i.e., immiscible with no interfacial tension or miscible with no diffusion), we used two contrasting numerical models for the simulations. The first model is of an immiscible interface with no interfacial tension and with the suspended region assumed to be a continuous fluid with apparent properties. The second is a point-force model that corresponds to a miscible interface with no diffusion. In this model, we assume each particle has no volume and we calculate the motion of the particles relative to 
the surrounding fluid. The results of both simulations are compared with previous experimental results. The role of the concentration interface on the collectivity of suspended particles is discussed from various viewpoints.

\section{Numerical simulations}

We treat a stratified suspension system in which a particle suspension is set on a pure fluid, as shown in Fig. 1. However, the liquid in the suspension is the same as pure fluid, so the system has no distinct interface. The concentration of particles, however, has a steep gradient at the apparent interface. We made the following two simulations: (1) a Lagrangian tracking of individual particles with two-way coupling to represent a miscible interface with no diffusion, and (2) an interface tracking of two immiscible continuum phases with no interfacial tension to verify whether the concentration interface behaves like a two-fluid interface by comparing the results with those of the Lagrangian-tracking simulation. Pan et al. (2001) simulated the RayleighTaylor instability of a suspension for two-dimensional cylindrical particles. Chou et al. (2014) also simulated the Rayleigh-Taylor instability of a suspension by using a two-fluid model based on complex constitutive equations. In this study, we treat the fully three-dimensional case and our simulations use a simple point-force model and a simple interface tracking model. The particle tracking approach treats particles in suspension individually. On the other hand, the interface tracking is an Euler-Euler approach with no diffusion. Our objective is to understand microscopically the dual nature of the concentration interface. To tackle this issue, we used both the particle tracking and the interface-tracking approaches. 


\subsection{Lagrangian tracking}

Consider particle motion with very low particle Reynolds number $R e \equiv$ $d_{\mathrm{p}} U_{\text {rel }} \rho / \mu$ (where $d_{\mathrm{p}}$ is the particle diameter, $U_{\text {rel }}$ is the particle velocity relative to fluid, $\rho$ is the fluid density, and $\mu$ is the fluid viscosity). As outlined by Bosse et al. (2005), we represent this scenario by using Stokes drag and gravity as:

$$
m_{\mathrm{p}} \frac{\mathrm{d} \boldsymbol{v}}{\mathrm{d} t}=-3 \pi \mu d_{\mathrm{p}}(\boldsymbol{v}-\boldsymbol{u}(\boldsymbol{y}))+m_{\mathrm{p}} \boldsymbol{g}\left(1-\frac{\rho}{\rho_{\mathrm{p}}}\right),
$$

where $\boldsymbol{v}$ and $\boldsymbol{u}$ are the particle velocity and fluid velocity, respectively, at particle position $\boldsymbol{y}, \boldsymbol{g}$ is the acceleration due to gravity, $m_{\mathrm{p}}$ is the particle $\operatorname{mass}\left[m_{\mathrm{p}}=V_{\mathrm{p}} \rho_{\mathrm{p}}, V_{\mathrm{p}}=(\pi / 6) d_{\mathrm{p}}^{3}\right]$, and $\rho_{\mathrm{p}}$ is the particle mass density. For a particle response time $\tau_{\mathrm{p}}$ that is much less than the characteristic timescale of the fluid,

$$
\tau_{\mathrm{p}}=\frac{m_{\mathrm{p}}}{3 \pi \mu d_{\mathrm{p}}}=\frac{d_{\mathrm{p}}^{2} \rho_{\mathrm{p}}}{18 \mu},
$$

the particle inertia can be neglected. By neglecting the inertia in Eq. (1), the instantaneous particle velocity is obtained from the fluid velocity at the particle position as

$$
\boldsymbol{v}=\boldsymbol{u}(\boldsymbol{y})+\tau_{\mathrm{p}} \boldsymbol{g}\left(1-\frac{\rho}{\rho_{\mathrm{p}}}\right) .
$$

In this case, the single-particle terminal velocity $U_{0}$ (i.e., the Stokes settling velocity) is $U_{0}=\tau_{\mathrm{p}} g\left(1-\rho / \rho_{\mathrm{p}}\right)$.

In this study, the particle volumetric concentration $\phi$ is as small as 0.02 or less. As done by Bosse et al. (2005), we treat the particle with the point-force model and consider the particle to have no volume. The fluid is assumed to be incompressible and its motion is described by the continuity equation

$$
\nabla \cdot \boldsymbol{u}=0
$$


and the two-way-coupled Navier-Stokes equation

$$
\frac{\partial \boldsymbol{u}}{\partial t}+\nabla \cdot \boldsymbol{u} \boldsymbol{u}=-\frac{1}{\rho} \nabla p+\frac{\mu}{\rho} \nabla^{2} \boldsymbol{u}+\frac{1}{\rho} \boldsymbol{f},
$$

where $\boldsymbol{f}$ is the feedback force per unit volume as given by

$$
\boldsymbol{f}(\boldsymbol{x})=-\frac{3 \pi \mu d_{\mathrm{p}}}{\Delta^{3}} \sum_{\boldsymbol{y}}(\boldsymbol{u}(\boldsymbol{y})-\boldsymbol{v}) w_{\mathrm{dst}}(\boldsymbol{x}-\boldsymbol{y}),
$$

where $\boldsymbol{x}$ is the grid-position and $\Delta$ is the grid spacing. The quantity $w_{\mathrm{dst}}$ is a nondimensional weighting function, and the trilinear distribution to eight neighboring points is given by $w_{\mathrm{dst}}$ as outlined in Bosse et al. (2005). The fluid-flow field is obtained by solving Eqs. (4) and (5) with Eq. (6). Equations (4) and (5) are discretized in space by a second-order central finite-difference method on a staggered-grid system. A second-order AdamsBashforth method is applied to advance the advection term in time, and a second-order Crank-Nicolson scheme is used for the viscous term. Pressure is linked to Eq. (4) by the simplified marker and cell (SMAC) algorithm (Amsden and Harlow, 1970) and the Poisson equation for pressure correction is solved by using a biconjugate gradient stabilized method (Ferziger and Perić, 1996). Fluid velocity at the particle position is interpolated from the surrounding grid points, and the particle position is updated by integrating Eq. (3) by using the second-order Adams-Bashforth method. In this study, the fluid velocity is interpolated based on a weighted average as follows:

$$
\boldsymbol{u}(\boldsymbol{y})=\frac{\sum_{\boldsymbol{x}} \boldsymbol{u}(\boldsymbol{x}) w_{\mathrm{int}}(\boldsymbol{x}-\boldsymbol{y})}{\sum_{\boldsymbol{x}} w_{\mathrm{int}}(\boldsymbol{x}-\boldsymbol{y})} .
$$

For the weight function $w_{\text {int }}$, we use the approximate delta function, which is similar to the approach of the immersed boundary method (Peskin, 2002):

$$
w_{\text {int }}(\boldsymbol{r})=w_{\text {int1D }}\left(r_{x}\right) w_{\text {int1D }}\left(r_{y}\right) w_{\text {int1D }}\left(r_{z}\right),
$$


where

$$
w_{\text {int } 1 \mathrm{D}}(r)=\left\{\begin{array}{ll}
1+\cos \frac{\pi r}{l_{\text {int }}}, & |r|<l_{\text {int }} \\
0, & |r| \geq l_{\text {int }}
\end{array} .\right.
$$

In Section 3.2, the region size $l_{\text {int }}$ is verified and discussed.

\subsection{Interface tracking}

To simulate the immiscible suspension and pure fluid, the front-tracking method (Shin and Juric, 2002; Yamamoto and Uemura, 2011) is used as interface-tracking method. In the front-tracking method, the interface between two fluids is tracked by marker points. The unit normal of the interface is calculated based on the triangle element connecting the marker points. By integrating the unit normals distributed over the grid points near the interface, we obtain the indicator function $I$, where $I=0$ represents the pure fluid phase and $I=1$ represents the suspension phase. Density and viscosity can be expressed by using the indicator function and the properties of each phase. With this information, we solve the continuity equation and the Navier-Stokes equation with variable density and viscosity by using the finite-difference method. The same scheme is used to solve the fluid equation as is used to solve the aforementioned Lagrangian tracking. The apparent density and viscosity of the suspension phase are given using volume fraction $\phi$ as,

$$
\begin{gathered}
\rho_{\text {sus }}=\phi \rho_{\mathrm{p}}+(1-\phi) \rho, \\
\mu_{\text {sus }}=(1+2.5 \phi) \mu .
\end{gathered}
$$

In the present study, we do not consider the interfacial tension force. In addition, density and viscosity is given by the inverse average and considering 
the distance from the interface, as proposed by Ferziger (2003) when the control volume or finite-difference points exist across the interface represented by $I=0.5$.

\section{Results and discussion}

\subsection{Simulation conditions}

The configuration of the present simulation is shown in Fig. 1. A noslip condition is imposed for each outer boundary. The height of the vessel $L=120 \mathrm{~mm}$, the width $W=100 \mathrm{~mm}$, the depth $D=8 \mathrm{~mm}$, and the suspension height $L_{\mathrm{sus}}=19.5 \mathrm{~mm}$; these dimensions are similar to those of the experiments by Harada et al. (2012). According to Harada et al. (2013), the wavelength $\lambda$ of the fastest-growing perturbation (i.e., "finger") is estimated by using the channel depth $D$ for a large-aspect-ratio vessel according to $\lambda=2 \pi D / \sqrt{6(\sqrt{5}-1)} \simeq 2.3 D$. Thus, about five fingers are assumed to grow under the present conditions. According to Harada et al. (2012), collective or individual settling is regularized by the ratio $H$ of the average particles separation $l$ to the finger wavelength $\lambda: H=l / \lambda$. Thus, we run simulations for several different values of $H$. The physical properties of the particles and liquid are given in Table 1. We used solid particles which have a diameter in the range of tens of microns to millimeters with various densities in viscous liquid. For such conditions, the diffusivity (including hydrodynamic diffusion) of particles is obviously less significant compared to the settling convection as observed in previous studies (Blanchette and Bush, 2005). The values of $H$ in Table 1 are computed by using the numerically determined wavelength $\lambda$ from the linear-stability analysis for an immiscible 
two-fluid Rayleigh-Taylor problem with a narrow channel, as described in Harada et al. (2012). The growth rate of the fastest growing perturbation $n_{\text {theo }}$ is also obtained from the linear-stability analysis.

The Lagrangian tracking model following the equilibrium approximation (3) requires the Stokes number to be much smaller than 1 (Balachandar and Eaton, 2010). In the present study, the flow field is laminar and the fluid time scale used in the Stokes number is determined by particle settling, then we defined the Stokes number as $\tau_{\mathrm{p}} /\left(d_{\mathrm{p}} / U_{0}\right)$. In the larger diameter cases, we set the particle mass density close to the fluid density, so Stokes number is kept much smaller than 1 . The maximum Stokes number is $O\left(10^{-3}\right)$ in case F.

The interface-tracking simulation uses neither the particle diameter $d_{\mathrm{p}}$ nor the number of particles $n_{\mathrm{p}}$. Thus, the average particle interval $l$ is not defined, nor $H$. In this study, the particle mass density for interface-tracking simulations is the same as used for cases A02 and A001 in Table 1. The ratio $H$ is given in terms of the unknown $d_{\mathrm{p}}$ as

$$
H=\left(\frac{\pi}{6 \phi}\right)^{\frac{1}{3}} \frac{d_{\mathrm{p}}}{\lambda},
$$

where $\lambda$ can be calculated without knowing $d_{\mathrm{p}}$ or $n_{\mathrm{p}}$ because of the continuum assumption. Thus, $H$ is proportional to $d_{\mathrm{p}}$ under the conditions of constant $\phi, \rho_{\mathrm{p}}$, and fluid properties.

Because of the nature of the diffusion equation, a larger viscosity leads to a smaller time step. To shorten the simulation time, the viscosity is set to two orders of magnitude less than that used in experiments. However, the Reynolds number used in the simulation is still sufficiently small $<O\left(10^{-1}\right)$, so only the timescale differs from experiments. 
The initial fluid and particle velocities are set to zero. For Lagrangian tracking, the initial particle positions are random. For interface tracking, the initial interface position is perturbed by a random amplitude $a_{k_{x}, k_{y}}$ for each integer wave numbers $\left(k_{x}, k_{y}\right)$ in the width $(x)$ and depth $(y)$ according to

$$
h(x, y)=\sum_{k_{x}} \sum_{k_{y}} a_{k_{x}, k_{y}} \sin \frac{\pi k_{x} x}{W} \sin \frac{\pi k_{y} y}{D} .
$$

The sums run from $-N_{x}+1$ to $N_{x}-1$ for $k_{x}$ and from $-N_{y}+1$ to $N_{y}-1$ for $k_{y}$, where $N_{x}$ and $N_{y}$ are the number of grid points in the $x$ and $y$ directions, respectively. The root mean square of the initial perturbation $h$ is set to $2 \Delta$. For simplicity, interface tracking just treats the lower interface of the suspension, so the upper half of the computational domain is simply filled with suspension.

\subsection{Verify region size used in weight function for Lagrangian simulation}

Several detailed studies referenced in Bosse et al. (2005) discuss how to interpolate the fluid velocity at the particle position, and an accurate interpolation scheme is given for the case in which particles experience turbulence or undergo many-particle interactions. However, for the low number density considered in the present study, only few-particle interactions are relevant, so reference grid points are more important than the computational accuracy. Simply speaking, the relative velocity used to compute the Stokes drag for a single particle falling in a stationary liquid is not the velocity field dragged by the target particle but the far field. Therefore, the fluid velocity at the particle position should be determined by the velocity field not including the effect of the target particle. However, taking only the far field is not suitable for the case in which other particles are present close to the target 
particle. Thus, we use the velocity averaged over a wide region that contains the target particle. To verify this approach, we simulate free-fall conditions for single particles in a square region with same depth $D=8 \mathrm{~mm}$ as used in the final simulations. Three-diameter particles $(0.05,0.5$, and $2.5 \mathrm{~mm})$ are tested with grid width $\Delta=D / 16=0.5 \mathrm{~mm}$. We test two initial positions for the particle center: one in which the particle falls through the cell center and one in which the particle falls along the cell ridge. Figure 2 shows the settling velocity error as a function of the size $l_{\text {int }}$ of the interpolation region. The results show that a small region leads to a large error and a large region leads to a small error. As seen in Fig. 2, the region size $2 l_{\text {int }}$ normalized by the gird size $\Delta$ and particle diameter $d_{\mathrm{p}}\left[2 l_{\text {int }} /\left(\Delta d_{\mathrm{p}}\right)^{1 / 2}>10\right]$ reduces the relative error to less than $10 \%$ regardless of the particle size and of the position relative to computational cell. Thus, for all results reported hereafter, $l_{\text {int }}=5\left(\Delta d_{\mathrm{p}}\right)^{1 / 2}$.

In the point force model, particle volume is not considered and its diameter is presented only in the fluid resistance force. In the present condition, particle Reynolds number is very low, so the point force model can approximately represent the Stokes flow around the sphere with considered diameter. We confirmed that the flow field around the sphere can be approximately represented even with 5 times larger diameter than the grid size.

\subsection{Dynamics and classification of concentration interface}

Figures 3 and 4 show the dynamics of the concentration interface obtained by Lagrangian tracking (interface tracking) for several different values of $H$ with $\phi=0.02$. For Lagrangian tracking, finger-shaped apparent interfaces appear from A02 to D02, as is the case for interface tracking. The shapes 
of A02 in Figs. 3(a)-3(d) and Figs.4(a)-4(d) are very similar. The width of the each figure corresponds to the vessel width $W=100 \mathrm{~mm}$. About five fingers are found in $W$, so that the estimate $\lambda \simeq 2.3 D$ is almost realized. For E02 and F02 in particular, individual motion of particles is superior to the motion as continuum because of low number density, so the concentration interface is not distinct. Each particle has a velocity relative to the local fluid velocity so, without the fingering instability, the suspensions settle. Because the present simulations use a viscosity two orders of magnitude less than that used in experiments, entire suspension blocks unrelated to the fingering instability can settle. For interface tracking, the pure fluid cannot penetrate the suspension so such settling without volume exchange by interface wave propagation is not reproduced. For $\phi=0.001$ (results not shown), the results follow a similar tendency as for $\phi=0.02$.

With these visual results, the fingertips (local under most points) may be tracked, so we can examine the dynamics of the average finger length $z_{\mathrm{f}}$ for several fingers under a single set of conditions. As examples, the results for A02, F02 and interface tracking are shown in Fig.5. As seen in Fig.5(a), the fingers grow exponentially during the initial stage and attain a constant velocity in the later stage. We fit an exponential $z_{\mathrm{f}}=z_{0} \exp n t$ to the results in the region where the finger length is less than $2 \mathrm{~mm}$ [as done by Harada et al. (2012)] to obtain the growth rate $n$. Next, the linear function $z_{\mathrm{f}}=U t+z_{1}$ is fit in the later stage to obtain the finger settling velocity $U$. We can confirm that the finger growth rates and the finger settling speeds of A02 and interface tracking are almost the same.

Following Harada et al. (2012), we use $U$ and $n$ to classify the results. 
The case of individual particle settling corresponds to $U / U_{0} \rightarrow 1$ and fluidlike settling does $U / U_{0} \gg 1$. For fluid-like settling, the ratio of $n$ to the growth rate of the fastest growing perturbation $n_{\text {theo }}$ (derived from the linearstability analysis for immiscible two-phase flow) goes to unity (i.e., $n / n_{\text {theo }} \rightarrow$ 1). For individual-particle settling, however, $n / n_{\text {theo }} \neq 1$.

Figure $6\left(\right.$ a) shows $n / n_{\text {theo }}$ and $U / U_{0}$ obtained by Lagrangian tracking as a function of $H$, which is the average particle separation normalized by the theoretical fastest-growing wavelength. For both $\phi=0.02$ and $\phi=0.001$, the results for $n / n_{\text {theo }}$ are close to unity for $H \lesssim 0.03$, whereas $n / n_{\text {theo }}$ grows exponentially as $H$ increases for $H>0.03$. The ratio $U / U_{0}$ grows exponentially with decreasing $H$ for $H \lesssim 0.1$ and is near unity for $H>0.1$. For example, the settling velocity for a finger is 100 times larger than the single-particle settling velocity for A02 $(H=0.014)$.

In Fig. $6(\mathrm{~b}), n / n_{\text {theo }}$ and $U / U_{0}$ obtained by interface tracking are shown as lines for various values of the parameter $d_{\mathrm{p}}$. For constant $\phi, H$ is proportional to $d_{\mathrm{p}}$ as mentioned before and $U_{0}$ is proportional to $d_{\mathrm{p}}^{2}$, as found by using Eq.(2) with constant density. Thus, $U / U_{0}$ is assumed to be proportional to $H^{-2}$. In addition, $n_{\text {theo }}$ is also independent of $d_{\mathrm{p}}$, so $n / n_{\text {theo }}$ is constant independent of $H$. By comparing Fig. 6(b) with Fig. 6(a), the tendencies $U / U_{0} \propto H^{-2}$ and $n / n_{\text {theo }} \rightarrow 1$ obtained by the continuum approach well describe the data obtained by Lagrangian tracking for $H \lesssim 0.03$.

Figure $6(\mathrm{c})$ shows $n / n_{\text {theo }}$ and $U / U_{0}$ obtained by experimentally, which is to be compared to the results for Lagrangian tracking [Fig. 6(a)] and interface tracking [Fig. 6(b)]. The results for $n / n_{\text {theo }}$ tend to unity for $H \lesssim 0.03$ and grow exponentially with increasing $H$ for $H>0.03$. The 
results for $U / U_{0}$ grow exponentially with decreasing $H$ for $H \lesssim 0.1$ and are near unity for $H>0.1$. Thus, the Lagrangian-tracking simulations are consistent with the experimental results. Note that these tendencies depend on the volume fraction $\phi$. The volume fraction used in the experiments range from 0.01 to 0.05 , so the present results for $\phi=0.02$ are in better agreement. This agreement confirms that the present Lagrangian-tracking simulation, which neglects particle volume and considers the feedback force only from neighboring grid points, can reproduce the flow field produced by a swarm of particles. The results obtained by interface tracking are consistent with those obtained by Lagrangian tracking and by experiment for $H \lesssim 0.03$. As is obvious, interface tracking, which is based on the continuum assumption, cannot reproduce particle-like behavior for $H>0.03$.

As discussed by Harada et al. (2013), when approximating fingers as diskshaped blobs, the settling velocity is calculated by balancing gravity and drag forces according to $U \sim\left(\rho_{\text {sus }}-\rho\right) g \lambda D / \mu \sim \phi\left(\rho_{\mathrm{p}}-\rho\right) g \lambda^{2} / \mu$. Thus, by considering $U_{0} \sim\left(\rho_{\mathrm{p}}-\rho\right) g d_{\mathrm{p}}^{2} / \mu$, we obtain the relation $U / U_{0} \sim \phi \lambda^{2} / d_{\mathrm{p}}^{2}=$ $\left(\phi^{1 / 2} d_{\mathrm{p}} / \lambda\right)^{-2}$. The factor in brackets depends on the volume fraction $\phi$ and corresponds to $H$ multiplied by $\phi^{1 / 6}$. We then rearrange the results by using the modified dimensionless number $H_{\text {mod }}=H / \phi^{1 / 6}$, as shown in Fig.7. We find that almost all results, regardless of whether they come from experiments, Lagrangian-tracking simulations, or interface-tracking simulations, give similar relationships for $n / n_{\text {theo }}$ and $U / U_{0}$ with respect to $H_{\bmod }$ and predict fluid-like settling for $H_{\text {mod }} \lesssim 0.1$. Finger speed cannot be obtained before performing experiments or simulations, however, $H$ can be estimated in advance. The finger width needs to be determined for $H$, but in principle 
it can be estimated theoretically (Harada et al., 2012, 2013).

\subsection{Stream envelope near concentration interface}

Figure 8 shows the Lagrangian-tracking results for instantaneous streamlines on the channel center plane relative to the lower-most particle for $\phi=0.02$, which corresponds to the third column of Fig.3 (partially magnified image near the lower-most particle). For A02 to C02 (corresponding to $H \lesssim 0.05, H_{\text {mod }} \lesssim 0.1$ ), streamlines are found to perfectly avoid the particle cloud that constitutes the fingertip. In such cases, the apparent concentration interface seals the surrounding fluid, so the suspension and the pure fluid behaves like an immiscible two-phase flow. However, for E02 and F02 (corresponding to $H \gtrsim 0.3, H_{\text {mod }} \gtrsim 0.5$ ), fluid streams penetrate the particle clouds, resulting in no distinct interface. For D02 (corresponding to $H \sim 0.1$, $H_{\text {mod }} \sim 0.2$ ), fluid streams avoid the tip of the cloud, but penetrate a part of the cloud, so the concentration interface is not distinct.

Nitsche and Batchelor (1997), Machu et al. (2001), and Metzger et al. (2007) discussed the stream envelope around a suspension droplet (particle cloud). For a low-Reynolds-number suspension droplet, the surrounding fluid does not penetrate the particle cloud, and the could behaves like a droplet of immiscible fluid with no interfacial tension. Such collective behavior of particles and sealing of fluid can be expressed by swarms of Stokeslets as discussed in Nitsche and Batchelor (1997), Machu et al. (2001), and Metzger et al. (2007). Stokeslets represent the flow field induced by each particle, so sealing by a concentration interface may be maintained by a sufficient number of Stokeslets. However, a small number of Stokeslets cannot maintain sealing because particles have a velocity relative to the border of the cloud. Thus, 
the resolution of the concentration interface discretized by point forces does determine whether the behavior of particles is collective or individual. The dimensionless number $H$ or $H_{\text {mod }}$ expresses the resolution of point forces for the spatial-perturbation scale $\lambda$. In other words, the resolution of the concentration interface expresses the important characteristics that can seal the surrounding fluid by particle-induced flow, and $H_{\text {mod }}$ can predict the transition from particle-like to fluid-like behavior.

\section{Conclusion}

Lagrangian particle tracking and interface tracking models were used to numerically simulate the collective and individual settling of particles suspended in a liquid under gravitational instabilities. The numerical results obtained from the two models are consistent with experimental results. Our numerical results also reveal the sealing behavior of a concentration interface that is due to flow generated by each particle. Finally, we propose a modified dimensionless parameter to describe the transition from collective to individual settling of suspended particles.

\section{Acknowledgment}

This study was supported by JSPS KAKENHI Grants No. 23560178 and No. 26420095. The authors would like to thank Enago (www.enago.jp) for the English language review. 


\section{References}

Amsden, A. A., and Harlow, F. H., 1970. A simplified MAC technique for incompressible fluid flow calculations. J. Comput. Phys. 6, 322-325.

Balachandar, S. and Eaton, J. K., 2010. Turbulent dispersed multiphase flow. Annu. Rev. Fluid Mech. 42, 111-133.

Bergougnoux, L., Ghicini, S., Guazzelli, E. and Hinch, J., 2003. Spreading fronts and fluctuations in sedimentation. Phys. Fluids 15, 1875-1887.

Blanchette, F. and Bush, J. W. M., 2005. Particle concentration evolution and sedimentation-induced instabilities in a stably stratified environment. Phys. Fluids 17, 073302-1-11.

Bosse, T., Kleiser, L. and Härtel, C., 2005. Numerical simulation of finite Reynolds number suspension drops settling under gravity. Phys. Fluids 17, 037101-1-17.

Burns, P. and Meiburg, E., 2012. Sediment-laden fresh water above salt water: linear stability analysis. J. Fluid Mech. 691, 279-314.

Carey, S., 1997. Influence of convective sedimentation on the formation of widespread tephra fall layers in the deep sea. Geology 25, 839-842.

Chou, Y., Wu, F. and Shih, W., 2014. Toward numerical modeling of fine particle suspension using a two-way coupled Euler-Euler model: Part 2: Simulation of particle-induced Rayleigh-Taylor instability. Int. J. Multiphase Flow 64, 44-54. 
Fernandez, J., Kurowski, P., Limat, L. and Petitjeans, P., 2001. Wavelength selection of fingering instability inside Hele-Shaw cells. Phys. Fluids 13, $3120-3125$.

Ferziger, J. H., 2003. Interfacial transfer in Tryggvason's method. Int. J. Numer. Meth. Fluids 41, 551-560.

Ferziger, J. H. and Perić, M., 1996. Computational Methods for Fluid Dynamics, Springer-Verlag.

Harada, S., Mitsui, T. and Sato, K., 2012. Particle-like and fluid-like settling of a stratified suspension. Eur. Phys. J. E 35, 1-1-6.

Harada, S., Kondo, M., Watanabe, K., Shiotani, T. and Sato, K., 2013. Collective settling of fine particles in a narrow channel with arbitrary crosssection. Chem. Engng. Sci. 93, 307-312.

Hoyal, D. C. J. D., Bursik, M. I. and Atkinson, J. F., 1999. Settling-driven convection: A mechanism of sedimentation from stratified fluids. J. Geophys. Res. 104, 7953-7966.

Kuenen, PH. H., 1968. Settling convection and grain size analysis. J. Sediment. Petrol 38, 817-831.

Kynch, G. J., 1952. A theory of sedimentation. Trans. Faraday Soc. 48, 166176.

Machu, G., Meile, W., Nitsche, L. C. and Schaflinger, U., 2001. Coalescence, torus formation and breakup of sedimenting drops: experiments and computer simulations. J. Fluid Mech. 447, 299-336. 
McCool, W. W. and Parsons, J. D., 2004. Sedimentaion from buoyant finegrained suspensions. Cont. Shelf Res. 24, 1129-1142.

Metzger, B, Nicolas, M and Guazzelli, É., 2007. Falling clouds of particles in viscous fluids. J. Fluid Mech. 580, 283-301.

Michioka, H. and Sumita, I., 2005. Rayleigh-Taylor instability of a particle packed viscous fluid: Implications for a solidifying magma. Geophys. Res. Lett. 32, L03309-1-4.

Mucha, P. J. and Brenner, M. P., 2003. Diffusivities and front propagation in sedimentation. Phys. Fluids 15, 1305-1313.

Nitsche, J. M. and Batchelor, G. K., 1997. Break-up of a falling drop containing dispersed particles. J. Fluid Mech. 340, 161-175.

Pan, T. W., Joseph, D. D., Glowinski, R., 2001. Modelling Rayleigh-Taylor instability of a sedimenting suspension of several thousand circular particles in a direct numerical simulation. J. Fluid Mech. 434, 23-37.

Parsons, J. D., Bush, J. W. M. and Syvitski, J. P. M., 2001. Hyperpycnal plume formation from riverine outflows with small sediment concentrations. Sedimentology 48, 465-478.

Pedley, T. J. and Kessler, J. O., 1992. Hydrodynaimc phenomena in suspensions of swimming microorganisms. Annu. Rev. Fluid Mech. 24, 313-358.

Peskin, C. S., 2002. The immersed boundary method. Acta Numerica 11, $479-517$. 
Shin, S. and Juric, D., 2002. Modeling three-dimensional multiphase flow using a level contour reconstruction method for front tracking without connectivity. J. Comput. Phys. 180, 427-470.

Snyder, P. J. and Hsu, T.-J., 2011. A numerical investigation of convective sedimentation. J. Geophys. Res. 116, C09024-1-15.

Völtz, C., Pesch, W. and Rehberg, I., 2001. Rayleigh-Taylor instability in a sedimenting suspension. Phys. Rev. E 65, 011404-1-7.

Völtz, C., 2003. Granular dynamics of density profiles in a suspension interface. Phys. Rev. E 68, 021408-1-5.

Warrick, J. A., Xu, J. Noble, M. A. and Lee, H. J., 2008. Rapid formation of hyperpycnal sediment gravity currents offshore of a semi-arid California river. Cont. Shelf Res. 28, 991-1009.

Yamamoto, Y. and Uemura, T., 2011. Numerical simulation of multiphase flows by level contour reconstruction method (improvement of volume conservation property). Trans. Japan Soc. Mech. Eng. 77, 237-246 (in Japanese).

Yu, X., Hsu, T.-J. and Balachandar, S., 2013. Convective instability in sedimentation: Linear stability analysis. J. Geophys. Res. Oceans 118, 256272 . 
Table 1: Physical properties of particles and fluid for Lagrangian-tracking simulations.

(a) $\phi=0.02\left(\rho=972 \mathrm{~kg} / \mathrm{m}^{3}, \mu=19.44 \mathrm{mPa} \mathrm{s}\right)$.

\begin{tabular}{cccccccc}
\hline Case & $\begin{array}{c}d_{\mathrm{p}} \\
{[\mu \mathrm{m}]}\end{array}$ & $\begin{array}{c}\rho_{\mathrm{p}} \\
{\left[\mathrm{kg} / \mathrm{m}^{3}\right]}\end{array}$ & $\begin{array}{c}n_{\mathrm{p}} \\
{[-]}\end{array}$ & $\begin{array}{c}U_{0} \\
{[\mu \mathrm{m} / \mathrm{s}]}\end{array}$ & $\begin{array}{c}\lambda \\
{[\mathrm{mm}]}\end{array}$ & $\begin{array}{c}n_{\text {theo }} \\
{\left[\mathrm{s}^{-1}\right]}\end{array}$ & $\begin{array}{c}H \\
{[-]}\end{array}$ \\
\hline$(\mathrm{A} 02)$ & 50 & 2500 & 4767009 & 107 & 13.1 & 3.71 & 0.0140 \\
$(\mathrm{~B} 02)$ & 150 & 1050 & 176556 & 49.2 & 17.9 & 0.258 & 0.0309 \\
$(\mathrm{C} 02)$ & 250 & 1050 & 38136 & 137 & 17.9 & 0.258 & 0.0515 \\
$(\mathrm{D} 02)$ & 500 & 1050 & 4767 & 547 & 17.9 & 0.258 & 0.103 \\
$(\mathrm{E} 02)$ & 1500 & 975 & 177 & 189 & 18.4 & 0.0102 & 0.299 \\
$(\mathrm{~F} 02)$ & 2500 & 973 & 38 & 175 & 18.5 & 0.00341 & 0.499 \\
\hline
\end{tabular}

(b) $\phi=0.001$ (fluid density and viscosity are same as (a)).

\begin{tabular}{cccccccc}
\hline Case & $d_{\mathrm{p}}$ & $\rho_{\mathrm{p}}$ & $n_{\mathrm{p}}$ & $U_{0}$ & $\lambda$ & $n_{\text {theo }}$ & $H$ \\
& {$[\mu \mathrm{m}]$} & {$\left[\mathrm{kg} / \mathrm{m}^{3}\right]$} & {$[-]$} & {$[\mu \mathrm{m} / \mathrm{s}]$} & {$[\mathrm{mm}]$} & {$\left[\mathrm{s}^{-1}\right]$} & {$[-]$} \\
\hline$(\mathrm{A} 001)$ & 20 & 6000 & 3724226 & 56.4 & 16.8 & 0.798 & 0.0119 \\
$(\mathrm{~B} 001)$ & 55 & 2000 & 179076 & 87.2 & 18.0 & 0.176 & 0.0305 \\
$(\mathrm{C} 001)$ & 90 & 2000 & 40869 & 233 & 18.0 & 0.176 & 0.0499 \\
$(\mathrm{D} 001)$ & 200 & 2000 & 3724 & 1150 & 18.0 & 0.176 & 0.111 \\
$(\mathrm{E} 001)$ & 550 & 1050 & 179 & 661 & 18.4 & 0.0136 & 0.299 \\
$(\mathrm{~F} 001)$ & 900 & 1050 & 41 & 1780 & 18.4 & 0.0136 & 0.489 \\
\hline
\end{tabular}




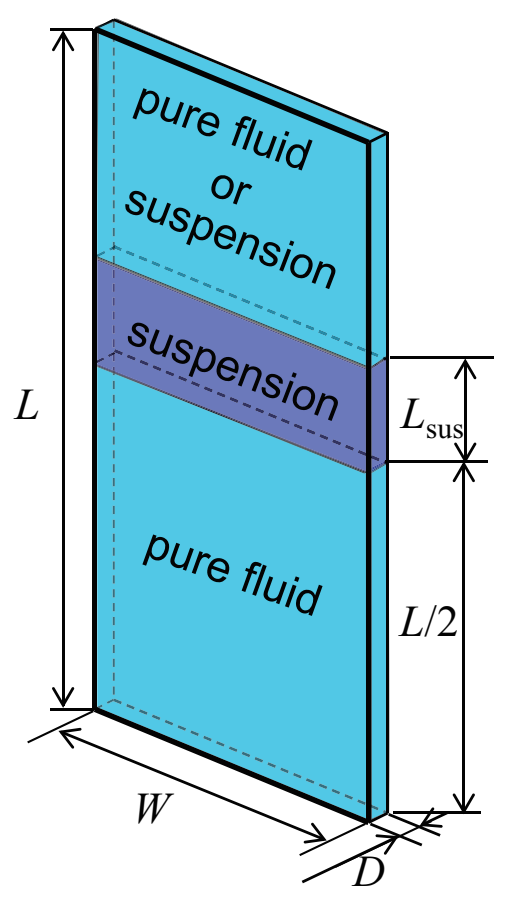

Figure 1: Configuration of simulations. Upper part is filled with suspension for interface tracking and pure fluid for Lagrangian particle tracking. 


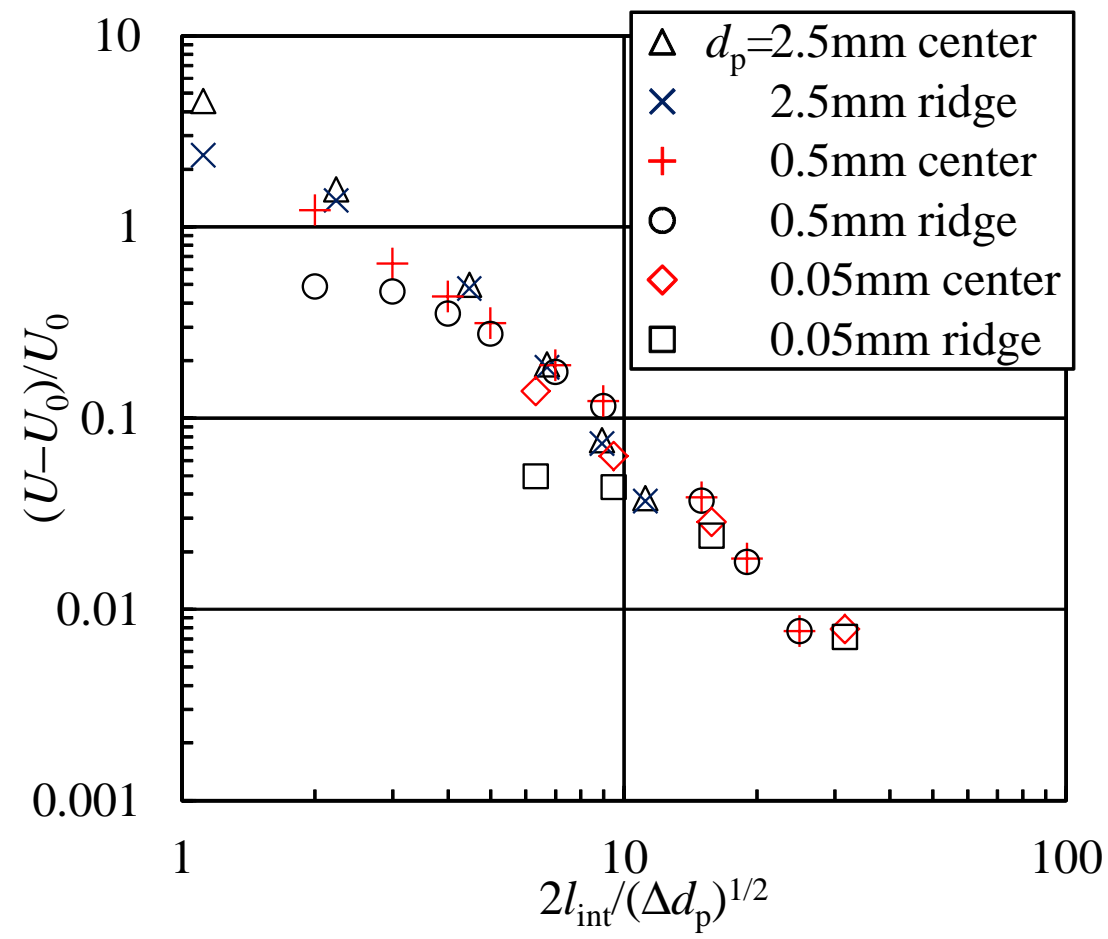

Figure 2: Effect of region size $l_{\text {int }}$ used in weight function $w_{\text {int }}$. $U$ is the computed settling velocity, $U_{0}$ is the Stokes settling velocity of a single particle, $\Delta$ is the grid width, and $d_{\mathrm{p}}$ is the particle diameter. 

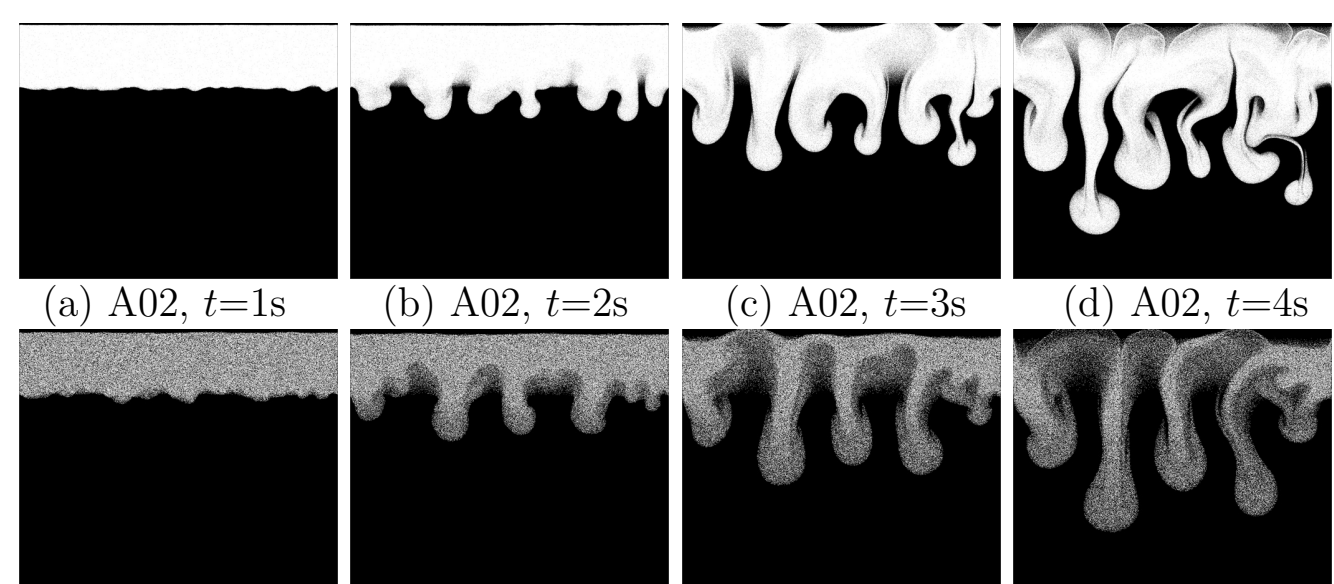

(b) $\mathrm{A} 02, t=2 \mathrm{~s}$

(c) $\mathrm{A} 02, t=3 \mathrm{~s}$

(d) $\mathrm{A} 02, t=4 \mathrm{~s}$
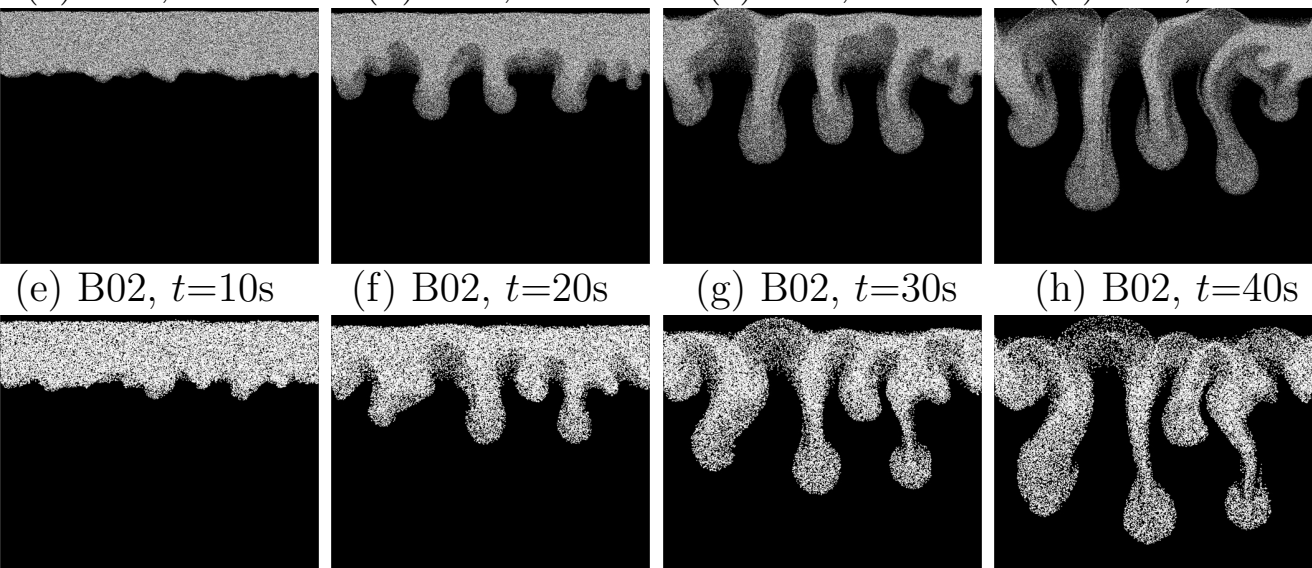

(f) $\mathrm{B} 02, t=20 \mathrm{~s}$

(g) B02, $t=30 \mathrm{~s}$

(h) B02, $t=40 \mathrm{~s}$
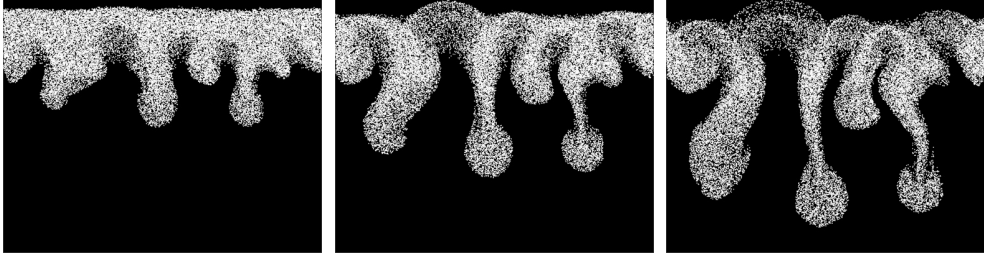

(i) $\mathrm{C} 02, t=10 \mathrm{~s}$

(j) $\mathrm{C} 02, t=20 \mathrm{~s}$

(k) $\mathrm{C} 02, t=30 \mathrm{~s}$

(l) $\mathrm{C} 02, t=40 \mathrm{~s}$

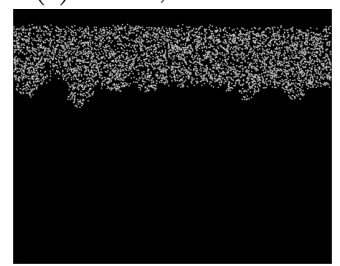

(m) D02, $t=8 \mathrm{~s}$
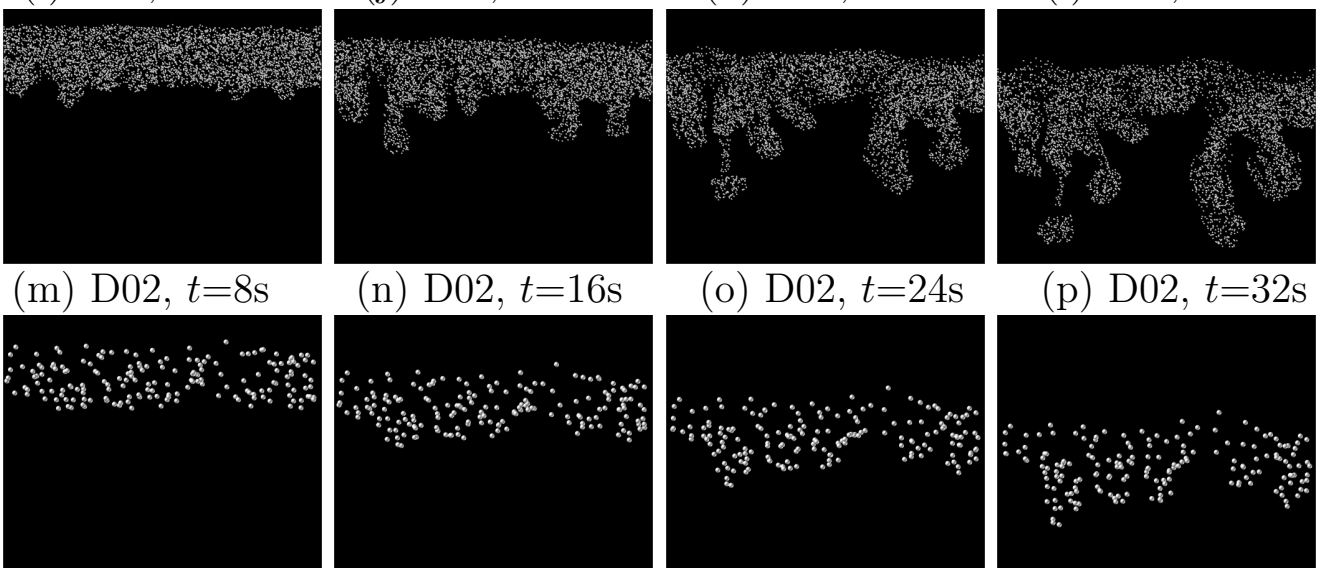

(q) E02, $t=40 \mathrm{~s}$

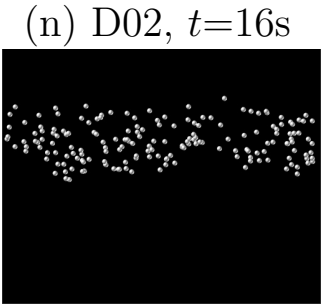

(o) $\mathrm{D} 02, t=24 \mathrm{~s}$

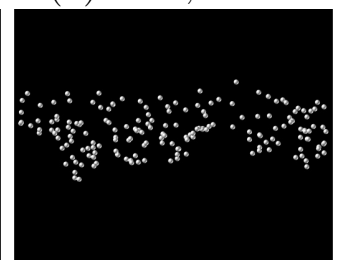

(p) D02, $t=32 \mathrm{~s}$

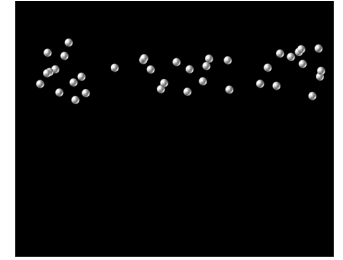

(u) F02, $t=50 \mathrm{~s}$

\begin{abstract}
(r) E02, $t=80 \mathrm{~s}$
\end{abstract}

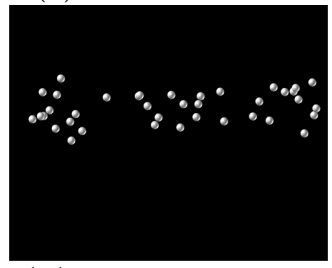

(v) F02, $t=100 \mathrm{~s}$ (s) E02, $t=120 \mathrm{~s}$

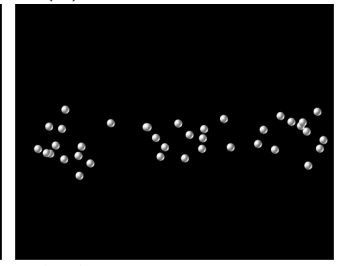

(w) F02, $t=150 \mathrm{~s}$

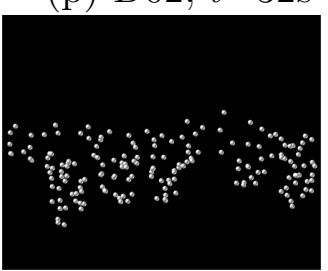

(t) $\mathrm{E} 02, t=160 \mathrm{~s}$

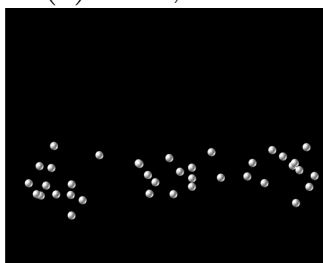

(x) F02, $t=200 \mathrm{~s}$

Figure 3: Snapshots of fingertip instability or particle-like settling obtained by Lagrangian tracking for $\phi=0.02$. 


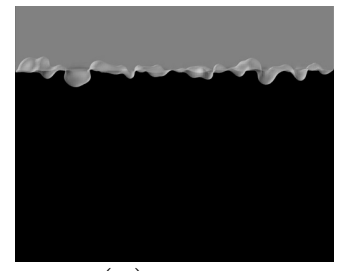

(a) $t=1 \mathrm{~s}$

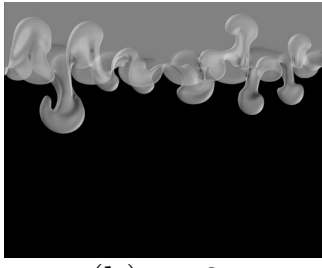

(b) $t=2 \mathrm{~s}$

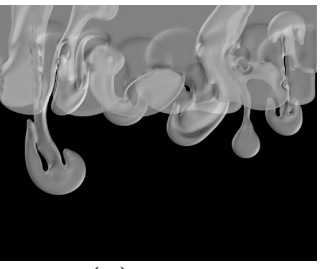

(c) $t=3 \mathrm{~s}$

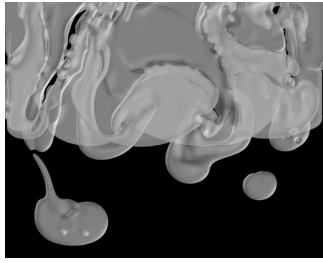

(d) $t=4 \mathrm{~s}$

Figure 4: Snapshots of fingertip instability obtained by interface tracking for $\phi=0.02$. 

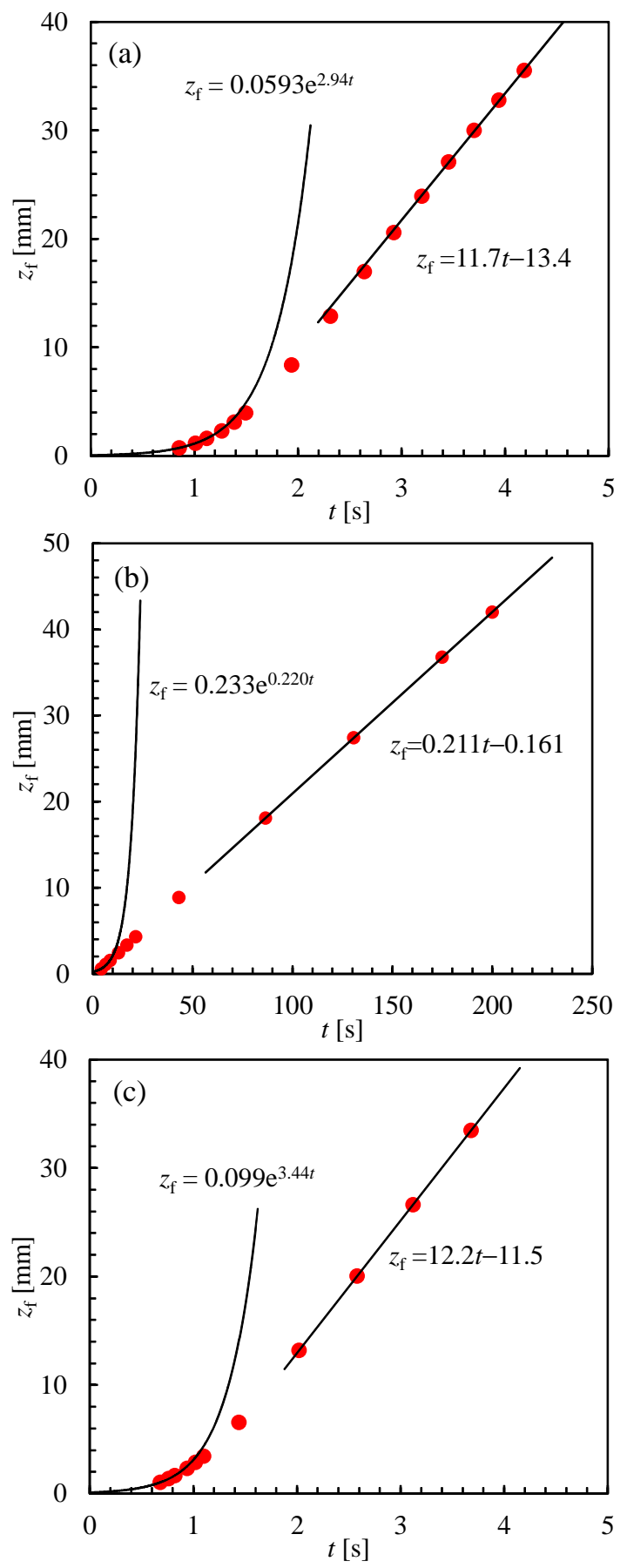

Figure 5: Temporal evolution of average finger length. (a) A02 ( $\phi=0.02, H=0.014)$, (b) F02 $(\phi=0.02, H=0.5),(c)$ interface tracking $(\phi=0.02)$. 

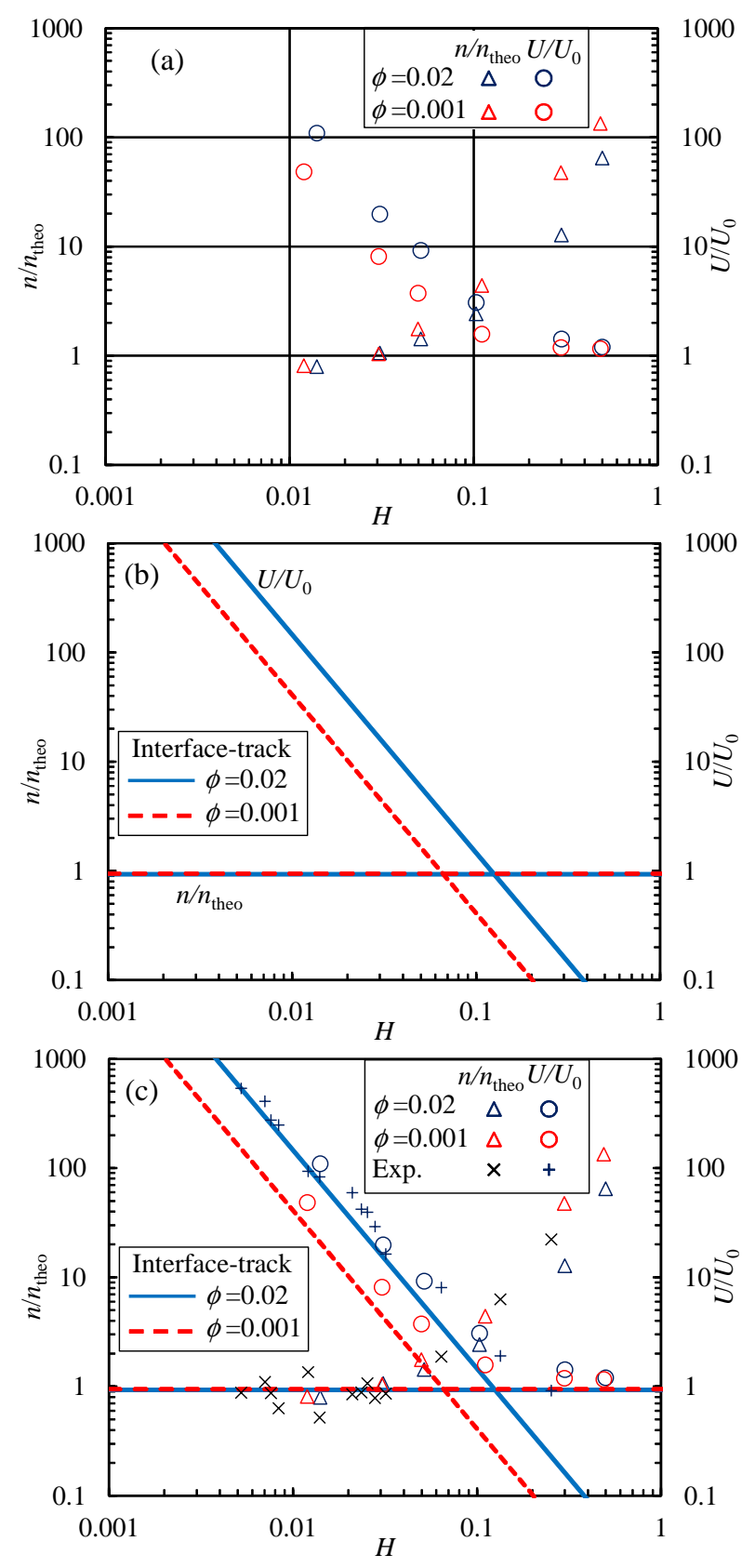

Figure 6: Classification of collective $\left(n / n_{\text {theo }} \rightarrow 1\right)$ or particle-like $\left(U / U_{0} \rightarrow 1\right)$ settling by dimensionless parameter $H$. (a) Results of Lagrangian tracking, (b) results of interfacetracking, and (c) results of both Lagrangian and interface tracking compared with experimental results taken from Harada et al. (2012). 


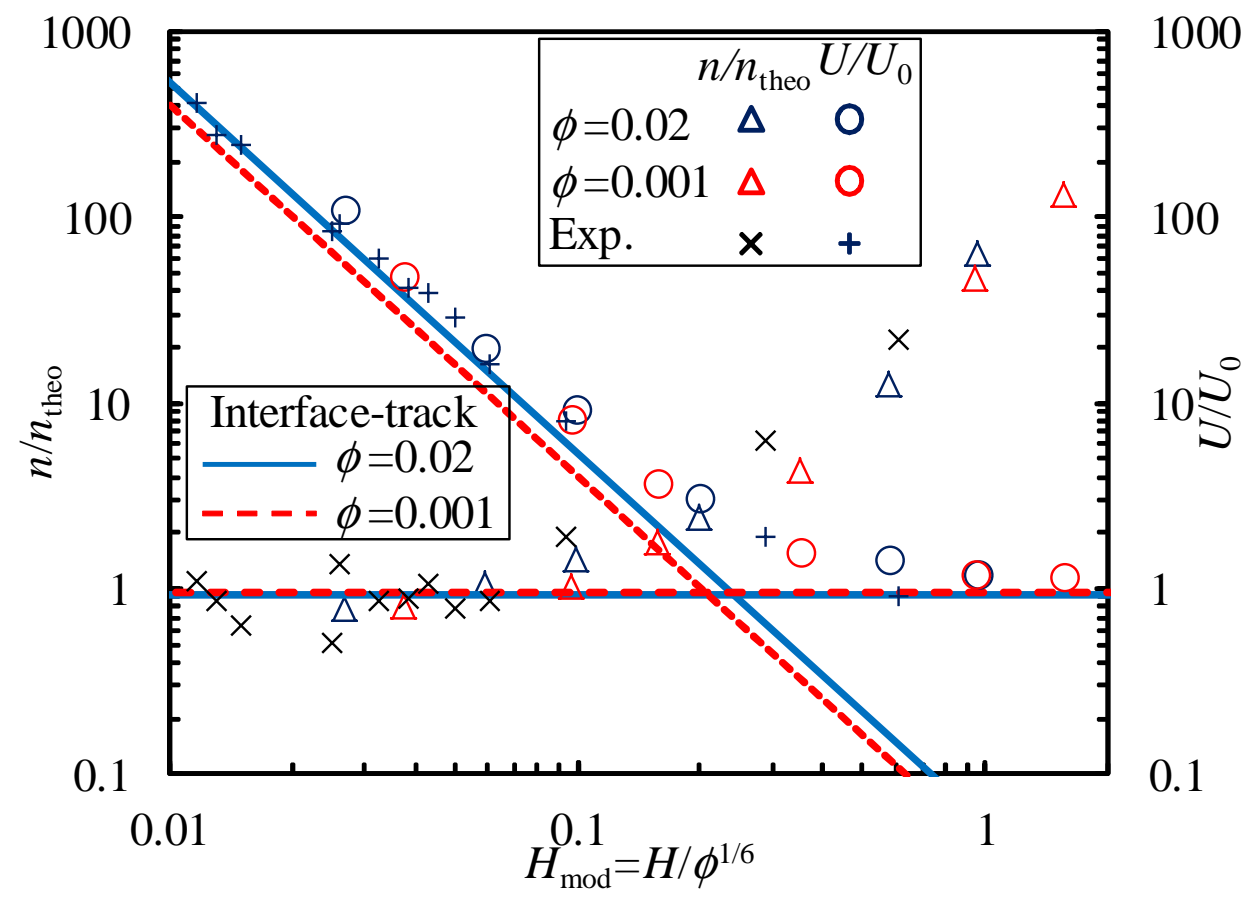

Figure 7: Classification of particle behavior as collective or individual settling based on modified dimensionless parameter $H_{\text {mod. }}$. Experimental data taken were from Harada et al. (2012). 


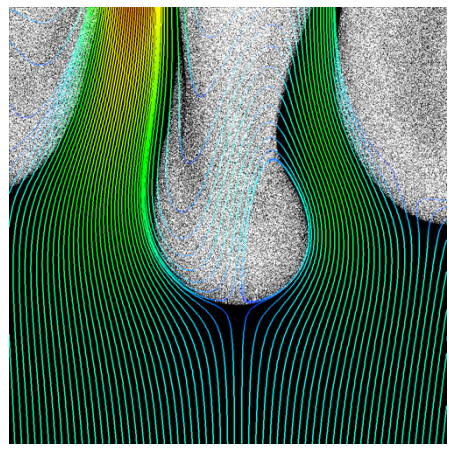

(a) $\mathrm{A} 02, t=3 \mathrm{~s}$

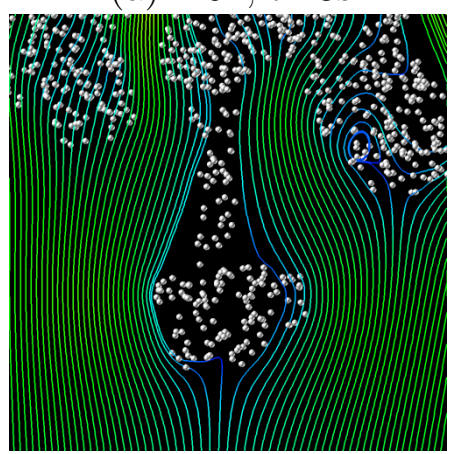

(d) D02, $t=24 \mathrm{~s}$

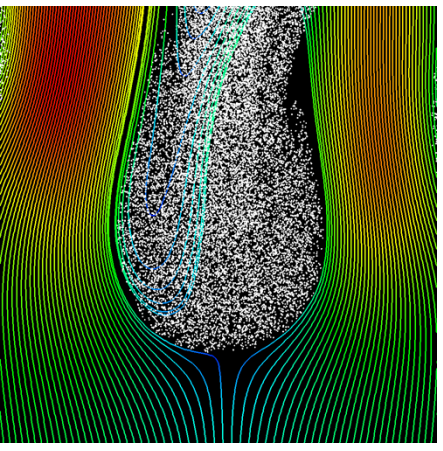

(b) B02, $t=30 \mathrm{~s}$

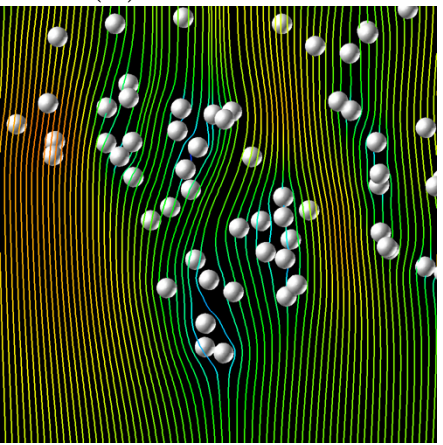

(e) $\mathrm{E} 02, t=120 \mathrm{~s}$

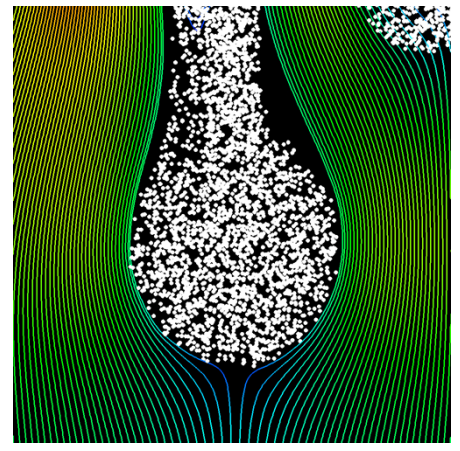

(c) $\mathrm{C} 02, t=30 \mathrm{~s}$

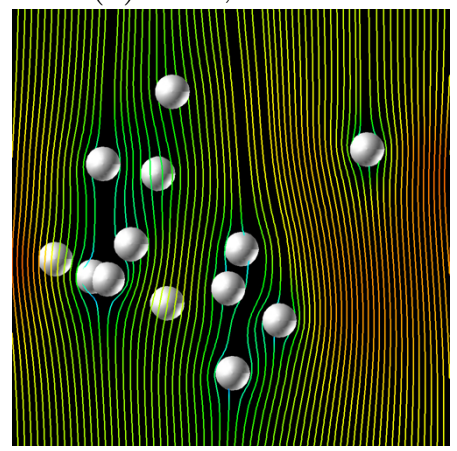

(f) $\mathrm{F} 02, t=150 \mathrm{~s}$

Figure 8: Instantaneous streamlines at the channel center plane relative to the lower-most particle obtained by Lagrangian tracking for $\phi=0.02$. Partly magnified images correspond to third columns in Fig. 3. 